\title{
Control of Local Electric Fields Influencing the Photoluminescence of an Individual CdTe/ZnTe Quantum Dot
}

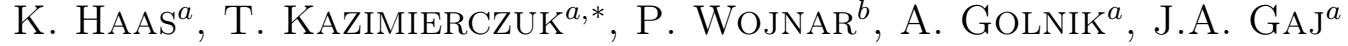 \\ AND P. KOSSACKI ${ }^{a, c}$ \\ ${ }^{a}$ Institute of Experimental Physics, University of Warsaw, Hoża 69, 00-681 Warsaw, Poland \\ ${ }^{b}$ Institute of Physics, Polish Academy of Sciences, al. Lotników 32/46, 02-668 Warsaw, Poland \\ ${ }^{c}$ Grenoble High Magnetic Field Laboratory, CNRS, B.P. 166, 38042 Grenoble Cedex 9, France
}

We examine the influence of a weak non-resonant illumination on the quantum dot photoluminescence spectrum. We observe that even very weak illumination affects both intensity and spectral position of emission lines in the spectrum. We discover no significant asymmetry in spatial dependence and infer that the observed effects cannot be attributed to a single neighbor center.

PACS numbers: 78.67.Hc, 78.55.Et

\section{Introduction}

Semiconductor quantum dots (QDs) are known to exhibit blinking - fluctuations of the spectral lines of individual QD emission, both in intensity and in the spectral position. These effects, occurring typically at non-resonant excitation, are usually explained by random variation of the charge state of the neighbor centers, leading to a randomly varying local electric field acting on the emitting dot. In typical experiments, where the blinking is observed, the light providing non-resonant excitation of the QD supplies also the carriers inducing the variation of the charge distribution in its neighborhood. To separate these two functions, we designed a two-color experiment: the QD is quasi-resonantly excited by a dye laser beam, while the free carriers influencing local electric fields are generated in the barrier by an Ar laser. In the present study we investigate a system of $\mathrm{CdTe} / \mathrm{ZnTe}$ self-organized quantum dots by the microphotoluminescence $(\mu \mathrm{PL})$ technique.

\section{Experimental}

The experiments were carried out on a sample containing self-assembled CdTe/ZnTe QDs grown by the tellurium desorption method [1]. The experiments were performed in a microphotoluminescence setup. The sample was mounted on a microscope objective [2] and placed inside a cryostat at $T=1.8 \mathrm{~K}$. Two $\mathrm{CW}$ lasers were

* corresponding author; e-mail: Tomasz.Kazimierczuk@fuw.edu.pl used to excite the photoluminescence. A tunable rhodamine 6G dye laser was used to excite QDs in quasi-resonant regime. Above barrier excitation was achieved with the use of $488 \mathrm{~nm}$ line from Ar ion laser. Intensity of both lasers was varied independently using neutral density filters. In case of quasi-resonant excitation, the exact wavelength was selected basing on measured photoluminescence excitation (PLE) spectra measured on studied QDs. Previous studies indicate that resonances present in PLE spectra are related to inter-dot excitation transfer [3], which was used as a convenient way to excite QD without generation of free carriers in the sample. Both lasers were focused on spots below $1 \mu \mathrm{m}$. Their positions were controlled independently.

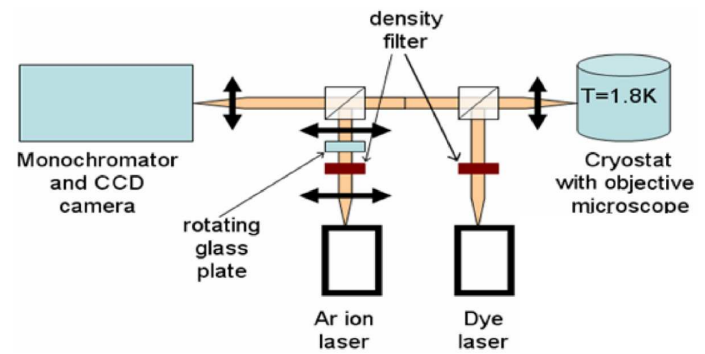

Fig. 1. Experimental setup used in the experiment.

A key element of the experiment was a scanning of the green laser spots positions. It was realized by rotating a $10 \mathrm{~mm}$ thick glass plate inserted in the convergent laser beam. Scanning of Ar ion laser spot did not affect excitation by the dye laser nor photoluminescence collection (Fig. 1). 


\section{Results}

A spectrally separated set of lines related to emission from a single QD was observed in the $\mu \mathrm{PL}$ spectrum. The lines in the spectrum followed a single dot emission pattern established in previous studies $[3,4]$, which allowed us to identify the main transitions as related to recombination of neutral exciton $(\mathrm{X})$, charged exciton $\left(\mathrm{X}^{-}\right)$, and biexciton $(\mathrm{XX})$. This identification was confirmed by polarization-resolved measurements of in-plane anisotropy of the QD.

A result of spatial mapping of the photoluminescence of a single QD with single exciting laser (Ar ion) is presented in Fig. 2. This measurement was used to estimate the laser spot diameter as being below $0.7 \mu \mathrm{m}$.

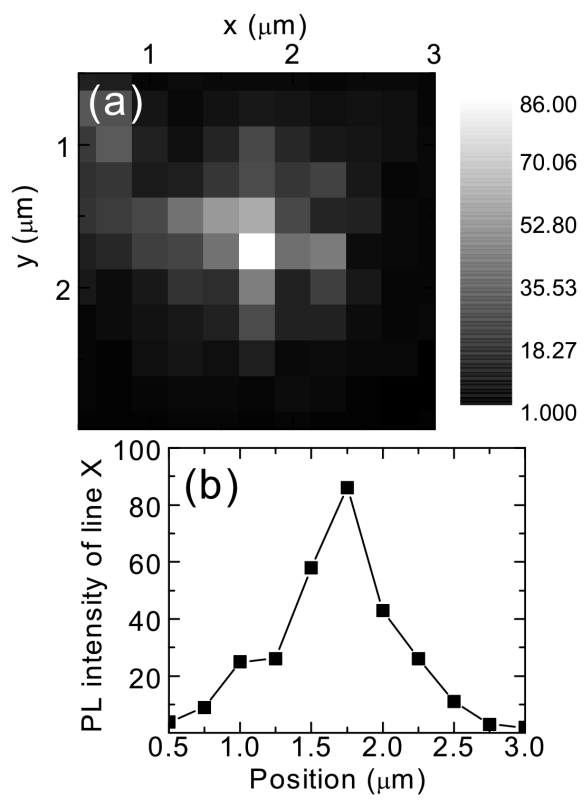

Fig. 2. (a) Mapping of the QD photoluminescence. The data was obtained using only Ar ion laser. Due to negligible QD size, the shape of the maximum is determined by size of the laser spot. (b) Cross-section of the map (a).

The second map (Fig. 3) presents results of two-color experiment. A dye laser $(40 \mu \mathrm{W})$ focused at fixed position was used as the main excitation channel. Simultaneously we scanned the vicinity of the QD by much weaker (up do $40 \mathrm{nW}$ ) above-barrier laser beam. This illumination was weak enough to make direct PL excitation negligible. On the other hand, the Ar laser illumination influenced both intensity and spectral position of the observed PL lines.

The intensity effect was observed on each line in the QD PL spectrum. The overall PL intensity of the QD increased by up to $25 \%$ under $40 \mathrm{nW}$ Ar illumination. The observed increase exceeded the PL intensity measured under excitation of the Ar laser alone by at least one order of magnitude. The intensity increase was accompanied by a pronounced blue-shift of the spectral lines.

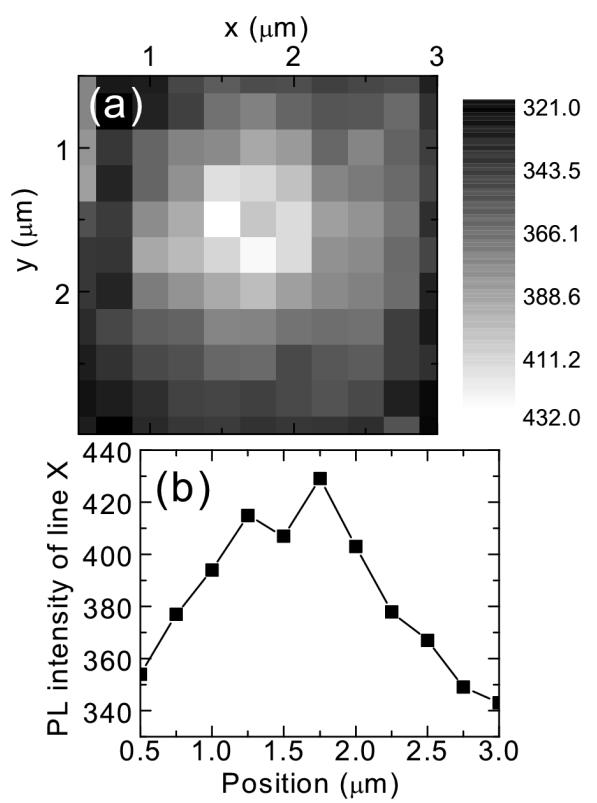

Fig. 3. (a) Mapping of the illumination influence on the QD photoluminescence. (b) Cross-section of the map (a).

The value of the blue-shift increased linearly with the Ar laser intensity and reached $0.4 \mathrm{meV}$ for $40 \mathrm{nW}$ Ar illumination. We attribute this blue-shift to the reduction of the Stark effect in the local carrier-induced electric field. Such reduction might be caused by the screening and/or center passivation by free carriers.

As expected, the observed effects vanished with increasing distance between the QD and the Ar laser spot. However, the range of the influence inferred from Fig. $3 \mathrm{~b}$ is noticeably larger than the laser spot size determined previously. We attribute tentatively this effect to the differences between diffusion properties of electrons and holes.

The recorded maps do not exhibit any significant directional asymmetry. Therefore, the obtained results do not support a hypothesis of a presence of a single neighbor center that would have a dominant contribution to local electric field and to blinking effect.

The spatial mapping of the above-barrier illumination in two-color experiment shows that even very weak illumination affects a PL spectrum of a QD. The obtained results do not support a hypothesis of a presence of a single neighbor center. However, we see clear signatures of quenching of local electric fields by free carriers created in the barriers.

\section{Acknowledgments}

This work was partially supported by the Polish Ministry of Science and Higher Education as research grants in years 2006-2010 and by European Project No. MTKD-CT-2005-029671. One of us (P.K.) acknowledges the 
support from European Project No. FP7/2007-2013-221515 (MOCNA).

\section{References}

[1] F. Tinjod, B. Gilles, S. Moehl, K. Kheng, H. Mariette, Appl. Phys. Lett. 82, 4340 (2003).

[2] J. Jasny, J. Sepiol, T. Irngartinger, M. Traber, A. Renn, U.P. Wild, Rev. Sci. Instrum. 67, 1425 (1996).

[3] T. Kazimierczuk, J. Suffczynski, P. Wojnar, A. Golnik, J.A. Gaj, P. Kossacki, Phys. Rev. B 79, 153301 (2009).
[4] J. Suffczyński, T. Kazimierczuk, M. Goryca, B. Piechal, A. Trajnerowicz, K. Kowalik, P. Kossacki, A. Golnik, K. Korona, M. Nawrocki, J.A. Gaj, G. Karczewski, Phys. Rev. B 74, 085319 (2006). 